\title{
Компенсация нелинейных искажений в системах с поляризационным мультиплексированием на основе полносвязанных нейронных сетей
}

\author{
С.А. Богданов $^{1, *}$, O.С. Сидельников $^{1}$, М.П. Федорук ${ }^{1,2}$, С.К. Турицын ${ }^{1,3}$ \\ ${ }^{1}$ Новосибирский государственный университет, г. Новосибирск \\ ${ }^{2}$ Институт вычислительных технологий СО РАН, г. Новосибирск \\ ${ }^{3}$ Астонский университет, Бирмингем, Великобритания \\ *E-mail: s.bogdanov@g.nsu.ru
}

DOI: 10.31868/RFL2020.124-125

Увеличение пропускной способности современных волоконно-оптических систем связи через повышение мощности сигнала (и увеличения отношения мощности сигнала к шуму, как в линейных каналах связи) затруднено ввиду нелинейности оптического волокона. Поляризационное уплотнение каналов (Polarization-division multiplexing - PDM), которое позволяет повысить скорость передачи данных вдвое за счет одновременной передачи информации по двум поляризационным компонентам волокна, приводит к дополнительным нелинейным искажениям из-за взаимодействия сигналов имеющих разные поляризации. Методы машинного обучения зарекомендовали себя как эффективный инструмент для цифровой обработки принятых сигналов в волоконно-оптических линиях связи $[1,2]$. В данной работе мы предлагаем использовать полносвязные нейронные сети (НC) прямого распространения для компенсации нелинейных искажений в приемнике систем передачи данных с поляризационным мультиплексированием. Были рассмотрены нейронные сети различной архитектуры и проведено сравнение их эффективности с линейной схемой компенсации.

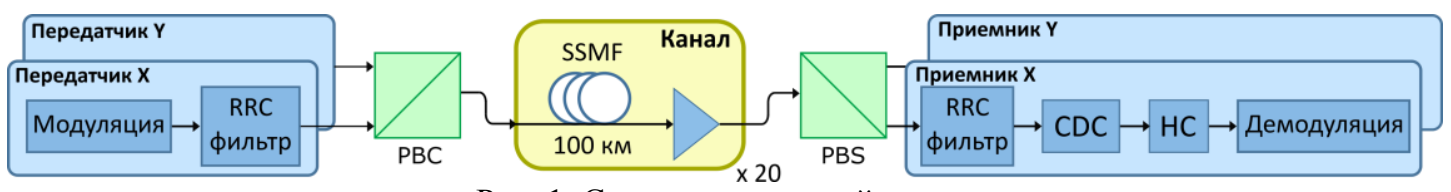

Рис. 1. Схема исследуемой линии

Схема исследуемой волоконной линии связи представлена на рисунке 1 . Каждый передатчик генерирует 16-QАМ сигнал с символьной скоростью $\mathrm{R}_{\mathrm{s}}=$ 32 Гбод. Для придания формы импульсам используется фильтр с характеристикой типа приподнятый косинус с коэффициентом сглаживания 0.1. Далее сигналы с разных передатчиков объединяются в PDM-сигнал, который затем подается в канал. Линия состоит из 20 пролетов стандартного одномодового волокна по 100 км каждый и эрбиевых оптических усилителей с $\mathrm{NF}=4.5$ дБ после каждого пролета. В приемнике после разделения поляризационных компонент сигнал проходит через согласованный фильтр, а затем выполняется идеальная компенсация хроматической дисперсии. Далее после понижения частоты дискретизации до 1 отсчета на символ происходит компенсации нелинейных эффектов с помощью предложенной схемы, на основе полносвязных нейронных сетей. Затем выполняется демодуляция сигнала и вычисление коэффициента битовых ошибок.

Для классификации 16-QАМ символов на приемнике используется нейронная сеть. Для обработки каждого символа на вход нейронной сети последовательно подаются действительные и мнимые части $\mathrm{N}$ предыдущих и $\mathrm{N}$ 
последующих символов с обеих поляризаций. Таким образом, входной слой состоит из $2 \cdot 2 \cdot(2 \cdot \mathrm{N}+1)$ нейронов. Сеть также имеет два скрытых слоя с варьируемым числом нейронов и нелинейными функциями активации (tanh). Выходной слой состоит из 16 нейронов, что соответствует количеству точек сигнального созвездия формата модуляции 16-QАМ. Для обучения нейронной сети использовался алгоритм оптимизации Adam библиотеки TensorFlow.

На рис. 2а показан результат применения нейронной сети на приемнике системы связи только с одной поляризацией и для случая, когда данные передавались по двум поляризациям, но на вход сети подавались символы одной из них (синяя и зеленая лини соответственно). В обоих случаях для каждого символа учитывались его 10 предыдущих и 10 последующих соседей, а сеть имела по 64 нейрона на скрытых слоях. Далее для системы с двумя поляризациями делается попытка учесть символы со второй поляризации для предсказания только первой (красные точки). Видно, что при 64 нейронах на скрытых слоях учет второй поляризации не дает никакого эффекта: это связано с тем, что такая простая сеть не способна обработать возросшее число символов на входе. И лишь увеличение числа нейронов на скрытых слоях позволяет повысить эффективность такой обработки сигнала за счет учета второй поляризации.

Рис. 26 показывает, как одна и та же нейронная сеть с 192 нейронами на каждом скрытом слое может обрабатывать данные с двух поляризаций в зависимости от числа используемых символов с каждой поляризации на входном слое. На графике также приведены результаты для линейной схемы на основе адаптивных фильтров (Least Mean Square - LMS), которая компенсирует нелинейный фазовый сдвиг. В данном случае нейронные сети предсказывают либо данные только с одной из двух поляризаций, либо сразу с двух (зеленые и красные точки соответственно). Видно некоторое ухудшение в эффективности сети для случая предсказания двух поляризаций, связанное с необходимостью разделения на 256 классов.
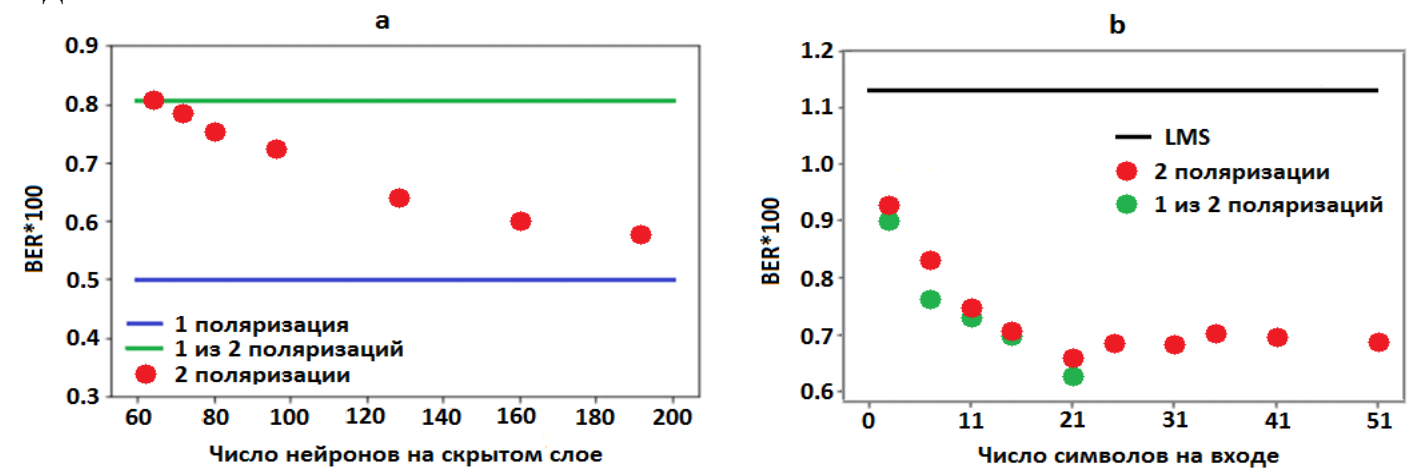

Рис. 2. Эффективность учета второй поляризации в зависимости от числа нейронов на скрытом слое (a). Результат применения нейронной сети для предсказания одной и двух поляризаций (b).

Работа Сидельникова О.С. (теоретический анализ) была выполнена при поддержке гранта Президента Российской Федерации (МК-915.2020.9). Работа Богданова С.А. (математическое моделирование) была поддержана государственным заданием на проведение фундаментальных исследований №FSUS-2020-0034.

\section{Литература}

[1] O.S. Sidelnikov et al, Opt.Express. 26, 32765-32776 (2018).

[2] C. Häger et al, Proc. OFC, W3A.4 (2018). 